\title{
Mortalidad atribuida al consumo de tabaco en España: Revisión sistemática
}

\section{Smoking-attributable mortality in Spain: A systematic review}

\author{
Julia Rey-Brandariz*, Mónica Pérez-Ríos***, Maria Isolina Santiago-Pérez***, \\ LeOnor Varela-Lema*,****, Alexandra Giraldo-Osorio*, *****, ******, Nerea Mourino*, \\ Alberto Ruano-Ravina *, **.
}

Este artículo forma parte de la tesis doctoral de Julia Rey Brandariz.

*Área de Medicina Preventiva y Salud Pública, Universidad de Santiago de Compostela, Santiago de Compostela, España. **CIBER de Epidemiología y Salud Pública, CIBERESP.

***Servicio de Epidemiología. Dirección General de Salud Pública. Xunta de Galicia, Santiago de Compostela, España.

****Unidad de Asesoramiento Científico-Técnico, Agencia Gallega de Conocimiento en Salud (avalia-t, ACIS), Santiago de Compostela, España.

*****Departamento de Salud Pública, Grupo de Investigación Promoción de la Salud y Prevención de la Enfermedad (GIPSPE), Universidad de Caldas, Manizales, Colombia.

******Fundación Carolina, Madrid, España.

\section{Resumen}

La mortalidad atribuida (MA) al consumo de tabaco es un indicador que refleja la evolución de la epidemia tabáquica a nivel poblacional. El objetivo de este trabajo es identificar y describir los estudios publicados que hayan estimado MA al consumo de tabaco en España. Se realizó una búsqueda en las bases de datos de PubMed y EMBASE de los trabajos publicados hasta el 15/04/2021. Se incluyeron estudios que estimaron MA en España en su conjunto o en unidades territoriales. Se identificaron 146 estudios y 22 cumplieron los criterios de elegibilidad. La primera estimación de MA en España data de 1978 y la última de 2017. En 12 estudios se estimó la MA a nivel nacional, 8 en comunidades autónomas, 1 a nivel provincial y 1 en una ciudad. La mayoría de estimaciones se realizaron en adultos mayores de 34 años categorizados como fumadores, exfumadores y nunca fumadores. La mortalidad observada derivó en todos los estudios de registros oficiales y los riesgos relativos mayoritariamente del Cancer Prevention Study II. En el periodo analizado se observó una disminución en la carga de MA en relación con la mortalidad total. En España se dispone de estimaciones de MA a nivel global, pero no tienen periodicidad regular y es infrecuente que se realicen en unidades territoriales. Debido a variaciones en la metodología y en las fuentes de datos es difícil evaluar de forma precisa cambios en la MA. Sería necesario disponer de estimaciones periódicas globales y regionales para monitorizar correctamente la epidemia tabáquica en España.

Palabras clave: mortalidad/fumar, tabaco, España, mortalidad.

\begin{abstract}
Smoking-attributable mortality (SAM) is an indicator that reflects the evolution of the tobacco epidemic at the population level. The objective of this study is to identify and to describe published studies that have estimated SAM in Spain. A search in PubMed and EMBASE databases was performed, limited to studies published until April $15^{\text {th }}$, 2021. Studies that estimated SAM in Spain or its constituent regions were included. Of the 146 studies identified, 22 met eligibility criteria. The first estimate of SAM in Spain dates from 1978 and the last from 2017. Twelve of the studies found estimated SAM at national level, 8 in regions, 1 in a province and 1 in a city. Most estimates were made for adults aged over 34, categorized as smokers, ex-smokers and never smokers. Observed mortality derived, in all studies, from official records, and relative risks mostly from Cancer Prevention Study II. In the period analyzed, a decrease in the burden of SAM was observed. In Spain, different SAM estimates are available globally, but they do not have regular periodicity, and such estimates are infrequently made by region. Due to variations in methodology and data sources, it is difficult to assess changes in SAM. Having global and regional periodic estimates would be necessary to correctly monitor the tobacco epidemic in Spain.

Key words: mortality/smoking, tobacco, Spain, mortality.
\end{abstract}

Recibido: Agosto 2020; Aceptado: Abril 2021.

Enviar correspondencia a: Mónica Pérez Ríos. Departamento de Medicina Preventiva y Salud Pública, Universidad de Santiago de Compostela, Santiago de Compostela, España. Telefono-Fax: 0034881812277

E-mail: monica.perez.rios@usc.es 
$\mathrm{E}$ 1 consumo de tabaco es la principal causa de muerte evitable a nivel mundial, debido a su elevada prevalencia y magnitud de asociación con diferentes causas de muerte (U.S. Department of Health and Human Services, 2014). En España, el 28,2\% de los hombres y el 20,8\% de las mujeres de 16 y más años eran fumadores en 2017 (Ministerio de Sanidad, Consumo y Bienestar, 2018). Según los datos del Instituto Nacional de Estadística (INE), en 2018 las enfermedades del sistema circulatorio, tumores y enfermedades respiratorias fueron las principales causas de muerte en España, todos ellos grandes grupos de causas de muerte asociados al consumo de tabaco (Instituto Nacional de Estadística, 2018).

Por ello, la Organización Mundial de la Salud (OMS) ha desarrollado estrategias como el MPOWER (Monitor, Protect, Offer, Warn, Enforce, Raise) y convenios como el "Convenio Marco de la OMS para el control del tabaco" con el fin de construir alianzas entre países que permitan elaborar políticas, movilizar recursos y planificar intervenciones para reducir el consumo de tabaco. Una de las estrategias implantadas en el MPOWER es la monitorización, entendida como la vigilancia de los indicadores que permitan describir la evolución de la epidemia tabáquica en la población (Organización Mundial de la Salud, 2008).

La epidemia tabáquica se puede monitorizar empleando diversos indicadores. Uno de ellos es la mortalidad atribuida (MA) al consumo de tabaco, que permite analizar de forma objetiva y sencilla, a partir de la aplicación de diferentes métodos de estimación, la evolución de la epidemia tabáquica y el impacto que genera sobre la salud de las poblaciones (Pérez-Ríos y Montes, 2008). Además, el cálculo de la MA permite comparar el impacto del tabaco entre zonas geográficas, grupos de edad o diferentes enfermedades relacionadas con su consumo.

En España, se han publicado estudios que estiman el impacto del tabaco en la mortalidad de la población. Estos estudios difieren en su alcance geográfico, fecha de publicación y metodología. Disponer de una revisión de toda la información disponible hasta el momento sobre el impacto del consumo de tabaco en la mortalidad de España y/o de sus unidades territoriales es fundamental para tener una visión completa de su impacto en la población española y para conocer cómo ha evolucionado a lo largo del tiempo. El objetivo de esta revisión es identificar y describir estos estudios.

\section{Métodos}

La metodología de búsqueda siguió las recomendaciones PRISMA ( Preferred Reporting Items for Systematic reviews and Meta-Analyses) (Page et al., 2021; Rethlefsen et al., 2021).

\section{Búsqueda bibliográfica}

Se realizó una búsqueda en las bases de datos MEDLINE (PubMed) y EMBASE utilizando como estrategia de bús- queda (( (mortality) AND attribut*) AND (smok* OR tobacco)) AND (Spain OR nombre de las comunidades y ciudades autónomas). En la base de datos EMBASE, la búsqueda se filtró por título, resumen y palabras clave. Los duplicados fueron eliminados manualmente. La búsqueda se completó con la revisión manual en metabuscadores como Google académico y con la revisión de la bibliografía de los registros que cumplieron con los criterios de selección.

La búsqueda se realizó el 1 de junio de 2020 y se actualizó el 15 de abril de 2021. Se configuró una alerta en PubMed para obtener actualizaciones semanales de nueva literatura hasta esa fecha. La revisión sistemática se registró en el sistema PROSPERO (Rey-Brandariz et al., 2021).

\section{Criterios de elegibilidad}

Se incluyeron todos los estudios que estimaron la MA al consumo de tabaco en España, con independencia del método de estimación empleado, en su conjunto o en unidades territoriales menores como comunidades autónomas (CCAA), provincia o ciudad publicados hasta el 15 de abril de 2021. No se incluyeron informes, comunicaciones a congresos, trabajos de simulación, trabajos que no realizaran estimaciones en población general, estimaciones conjuntas con otros factores de riesgo, reanálisis de MA, que estimarán exclusivamente años potenciales de vida perdidos (APVP), analizaran morbilidad o realizaran proyecciones. La búsqueda no se limitó por idioma.

\section{Selección de estudios y extracción de la información}

Dos investigadores (JRB y AGO) revisaron, de forma independiente, los títulos y resúmenes de los registros identificados. Se obtuvieron y leyeron a texto completo los registros preseleccionados por ambos. La información de los registros que cumplieron criterios de elegibilidad se extrajo en una base de datos. La información extraída de cada registro fue: año y revista de publicación; en relación con la MA: ámbito geográfico (lugar geográfico para el que se hizo la estimación), ámbito temporal (año/s de las estimaciones), edades a estudio, grupos de edad para los que se estimó la MA y método de estimación empleado; en relación con la prevalencia de consumo: fuente de datos, año del estudio del que deriva la prevalencia, categorías de consumo de tabaco empleadas (fumador, exfumador, nunca fumador) y grupos de edad; en relación a los riesgos relativos (RR): fuente de datos y en relación con la mortalidad observada (MO): fuente de datos, año de mortalidad y causas analizadas. Se extrajo información sobre el porcentaje de MA frente a la MO en global, en hombres y en mujeres; el grupo de causas de muerte (tumores, enfermedades cardiovasculares/cardiometabólicas y respiratorias) y la causa específica de muerte con mayor carga de MA al consumo de tabaco en ambos sexos, en hombres y en mujeres. Las diferencias en la extracción realizada por los dos investigadores (JRB y AGO) se resolvieron por consenso. 


\section{Resultados}

\section{Resultados de la búsqueda}

Se obtuvieron 146 registros de los cuales 22 cumplían con los criterios de elegibilidad. La búsqueda en EMBASE aportó 13 registros no identificados en la búsqueda en PubMed, de estos 13 registros uno cumplió con los criterios de inclusión. La revisión de la bibliografía de los estudios incluidos aportó dos registros y la búsqueda en Google académico no aportó ningún registro a mayores. Se identificaron 34 registros potencialmente relevantes y de estos se excluyeron 12 después de la lectura a texto completo. Los motivos de exclusión fueron que las estimaciones se realizaron en una población específica (población en edad de trabajar: 35-64 años) (Olivia-Moreno, Trapero-Bertran y
Peña-Longobardo, 2019), la estimación era conjunta con otros factores de riesgo (Janssen, Trias-Llimós y Kunst, 2021), realizaban un reanálisis de la MA calculada en anteriores estudios (Haeberer et al., 2020), no realizaban atribución de mortalidad (Gregoraci et al., 2017; Kulik et al., 2014; Long et al., 2021; Mackenbach et al., 2015, Rodríguez Tapioles, Pueyos Sánchez, Bueno Cavanillas, Delgado Rodríguez y Gálvez Vargas, 1994), estimaban APVP (García Benavides y Hernández Aguado, 1989), analizaban morbilidad (González-Enríquez et al., 2002;) o eran proyecciones (Banegas Banegas et al., 1993; Sánchez et al., 2010). El proceso de selección de registros aparece reflejado en la Figura 1, y en la Tabla 1 se describen las principales características de los estudios incluidos.

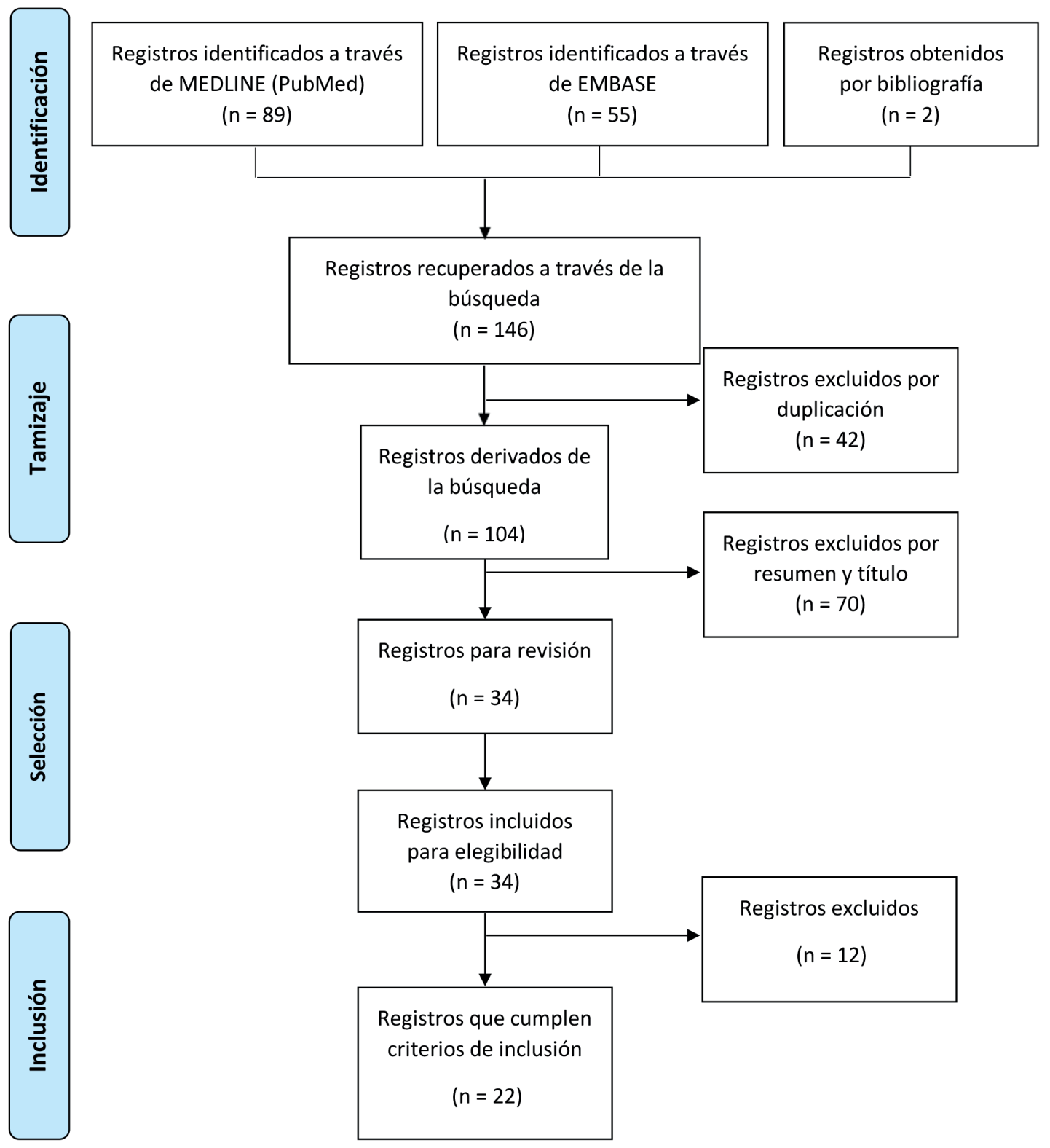

Figura 1. Flujograma de la búsqueda bibliográfica. 


\section{Características de los estudios incluidos}

En cuanto a los estudios incluidos, 12 realizaron estimaciones de MA a nivel nacional (Banegas et al., 2011; Banegas, Díez Gañán, González Enríquez, Villar Álvarez y Rodriguez-Artalejo, 2005; Banegas, Díez Gañán, Rodríguez-Artalejo, Pérez-Regadera y Villar Álvarez, 2001; Banegas, Rodríguez-Artalejo, Graciani, Billar y Herruzo, 2003; González Enríquez, Rodríguez Artalejo, Banegas Banegas y Villar Álvarez, 1989a; González Enríquez, Rodríguez Artalejo, Martín Moreno, Banegas Banegas y Villar Álvarez, 1989b; González Enríquez, Villar Álvarez, Banegas Banegas, Rodríguez Artalejo y Martín Moreno, 1997; Gutiérrez-Abejón et al., 2015; Hernández-García, Sáenz-González y González-Celador, 2010; Montes, Pérez-Ríos y Gestal, 2004; Pérez-Ríos et al., 2020; Rey et al., 2021), 8 en CCAA (Bello Luján, Lorenzo Ruano, Gil Muñoz, Saavedra Santana y Serra Majem, 2001; Criado-Álvarez, Morant Ginestar y De Lucas Veguillas, 2002; Pérez Ríos et al,. 2009; Pérez Ríos et al., 2011; Santana Armas, Orengo, Santana Armas, Lorenzo y Serra Majem, 1998; Santos Zarza, Valero Juan y Sáenz González, 2001; Valero Juan, Carrero Santos, Nelia Lubián y Sáenz González, 1999; Zorrilla-Torras, García-Marín, Galán-Labaca y Gandarillas-Grande, 2005), 1 a nivel provincial (Granada) (Rodríguez Tapioles et al., 1997) y 1 en una ciudad (Barcelona) (Jané, Borrell, Nebot y Pasarín, 2003). A nivel nacional, se realizaron estimaciones para el periodo 1978-1992 y en los años: 1983, 1984, 1998, 2012, 2016 y 2017 y en 2 ocasiones para 2001 y 2006. Galicia y las Islas Canarias son las CCAA con el mayor número de estimaciones de MA al consumo de tabaco.

El método dependiente de prevalencias se empleó para estimar la MA en todos los estudios, exceptuando un estudio realizado en Galicia en el que se utilizó el método independiente de prevalencias. En relación con la edad de la población a estudio, 15 análisis se realizaron en adultos de 35 años y más, 4 en población de 15/18 años y más y en 3 estudios no se indicó la edad. En 20 de los 21 estudios en los que se empleó el método dependiente de prevalencias, éstas procedían de encuestas nacionales o locales y en 15 se tuvieron en cuenta 3 categorías de consumo: fumadores, exfumadores y nunca fumadores. La MO derivó en todos los estudios de registros oficiales y en 16 de los 22 estudios, los RR derivaron del Cancer Prevention Study II (CPS-II) (Tabla 1).

En 15 estudios se estimó la MA para más causas de muerte que las asociadas causalmente al consumo de tabaco en el informe del Surgeon General de referencia para el año de la estimación y en 4 se atribuyó la mortalidad a las causas con relación causal establecida al consumo de tabaco en el momento de la estimación (Tabla 1).

En todos los estudios, la atribución de mortalidad se centró en el consumo de tabaco, excepto en dos estudios; uno realizado en Barcelona (Jané et al., 2003) en el que también se estimó la MA al consumo de alcohol y otro en
España en el que también se estimó MA a la tensión arterial, sobrepeso/obesidad y diabetes (Banegas et al., 2003).

\section{Resultados de la estimación de la MA al consumo de tabaco}

En el año 1978, se estimó que el 13,7\% de las muertes observadas a nivel nacional se atribuyeron al consumo de tabaco (González Enríquez et al., 1997), este porcentaje aumentó hasta un 16,0\% en 1998 (Banegas et al., 2001), se mantuvo en torno al 15\%-16\% hasta 2012 (Gutiérrez Abejón et al., 2015) y disminuyó hasta el 13,7\% en 2016 (Pérez Ríos et al., 2020). Por CCAA la proporción de MA frente a la MO en Castilla y León fue del 9,4\% en 1995 (Santos Zarza et al., 2001) y en Extremadura del 11,7\% en 1993 (Valero Juan et al., 1999). En la estimación realizada en 2017, la MA aumentó en ambas CCAA a un 11,3\% y 13,6\%, respectivamente (Rey et al., 2021). En las Islas Canarias el porcentaje de MA alcanzó el 20,7\% en 1975, descendió a 15,3\% en 1994 (Bello Luján et al., 2001) y se mantuvo estable en 2017 (15,3\%) (Rey et al., 2021). Las estimaciones para Castilla La Mancha fueron del 18,7\% en 1987 y 1997 (Criado Álvarez et al., 2002) y del 12,4\% en 2017 (Rey et al., 2021).

En hombres, se estimó que la MA al tabaco supuso el 20,3\% de la MO total en 1983 (González Enríquez et al., 1989a), aumentó al 28,3\% en 1998 (Banegas et al., 2001) y disminuyó a partir del 2001 (Banegas et al., 2005), hasta alcanzar el 22,6\% en 2012 (Gutiérrez Abejón et al., 2015). En mujeres, el porcentaje de MA al tabaco, frente a la MO total, fue de un 5,4\% en 1983 (González Enríquez et al., 1989a), se situó en torno al 2\%-3\% entre 1992-2001 (Banegas et al., 2001; Banegas et al., 2005; González Enríquez et al., 1997; Montes et al., 2004,) y aumentó al 7,8\% en 2006 (Hernández García et al., 2010). La MA en hombres fue descendiendo al avanzar el año a estudio; así, en 1998 era del 92,5\% (Banegas et al., 2001), i.e el 92,5\% de la MA al consumo de tabaco sucedía en los hombres, del 91,0\% en 2001 (Banegas et al., 2005; Montes et al., 2004); y del 84\%-85\% en 2016 y 2017 (Pérez Ríos et al., 2020; Rey et al., 2021).

El primer estudio que estimó la MA en España en 1978, estableció que el 51, 4\% de la MA al consumo de tabaco había sido por enfermedades cardiovasculares/cardiometabólicas, lo que las situó como el grupo de causas con mayor carga de MA (González Enríquez et al., 1997). Este porcentaje disminuyó al 30\%-35\% en las estimaciones realizadas entre 2001 y 2006 (Hernández-García et al., 2010; Montes et al., 2004), y al 27,5\% en la estimación realizada en 2017 (Rey et al., 2021). A partir de los años 90, los tumores se situaron como el principal gran grupo de causas de MA al consumo de tabaco, así en las últimas estimaciones el 50\% de la MA se debió a los tumores (Pérez-Ríos et al., 2020; Rey et al., 2021). La carga de MA a enfermedades respiratorias se ha mantenido estable entorno al 20\%-23\% desde las estimaciones en 1992 y 1998 (Banegas et al., 2001; Gon- 
Julia Rey-Brandariz, Mónica Pérez-Ríos, Maria Isolina Santiago-Pérez, Leonor Varela-Lema, Alexandra Giraldo-Osorio, Nerea Mourino, Alberto Ruano-Ravina

Tabla 1. Principales características de los estudios que estiman mortalidad atribuida (MA) al consumo de tabaco en España (nivel nacional, comunidad autónoma, provincia, ciudad), 1978-2017.

\begin{tabular}{|c|c|c|c|c|c|c|c|c|c|c|c|c|}
\hline \multirow[b]{2}{*}{ Autor } & \multirow[b]{2}{*}{ Ámbito } & \multirow{2}{*}{$\begin{array}{c}\text { Año } \\
\text { estimación } \\
\text { MA }\end{array}$} & \multirow{2}{*}{$\begin{array}{c}\text { Edad } \\
\text { población }\end{array}$} & \multirow{2}{*}{$\begin{array}{l}\text { Grupos de } \\
\text { edad de la } \\
\text { estimación }\end{array}$} & \multicolumn{3}{|c|}{ Prevalencia } & \multirow{2}{*}{$\begin{array}{c}\text { Riesgo Relativo } \\
\text { Fuente }\end{array}$} & \multicolumn{4}{|c|}{ Mortalidad observada } \\
\hline & & & & & Año & $\begin{array}{l}\text { Categorías } \\
\text { consumo }\end{array}$ & $\begin{array}{l}\text { Grupos } \\
\text { de edad }\end{array}$ & & Año & $A \tilde{o} \mathbf{S G} \phi$ & $\begin{array}{l}\text { Causas no } \\
\text { incluidasף }\end{array}$ & $\begin{array}{c}\text { Causas } \\
\text { adicionalesף }\end{array}$ \\
\hline \multicolumn{13}{|c|}{ NACIONAL } \\
\hline $\begin{array}{l}\text { González } \\
\text { Enríquez } \\
\text { et al., 1989a }\end{array}$ & España & 1983 & - & - & $\begin{array}{l}1970- \\
1979\end{array}$ & - & - & $\begin{array}{l}\text { Diferentes } \\
\text { estudios }\end{array}$ & 1983 & 1984 & - & Asma \\
\hline $\begin{array}{l}\text { González } \\
\text { Enríquez } \\
\text { et al., 1989b }\end{array}$ & España & 1984 & - & - & - & - & - & $\begin{array}{l}\text { Diferentes } \\
\text { estudios }\end{array}$ & 1984 & 1984 & - & Asma \\
\hline $\begin{array}{l}\text { González } \\
\text { Enríquez } \\
\text { et al., } 1997\end{array}$ & España & $\begin{array}{l}1978- \\
1992\end{array}$ & $\geq 35$ años & $\begin{array}{c}35-44 / 45-64 \\
/ \geq 65\end{array}$ & $\begin{array}{l}1978- \\
1992\end{array}$ & $\begin{array}{l}\text { Fumador, } \\
\text { exfumador, } \\
\text { nunca fumador }\end{array}$ & $\begin{array}{c}35-44 / 45- \\
64 / \geq 65\end{array}$ & CPS II & 1978-1992 & 1989 & $\begin{array}{c}\text { Otras } \\
\text { vascularest }\end{array}$ & $\begin{array}{c}\text { Enfermedad } \\
\text { hipertensiva, } \\
\text { neumonía e } \\
\text { influenza y asma }\end{array}$ \\
\hline $\begin{array}{l}\text { Banegas } \\
\text { et al., } 2001\end{array}$ & España & 1998 & $\geq 35$ años & $\begin{array}{c}\text { No } \\
\text { especifican }\end{array}$ & 1997 & $\begin{array}{l}\text { Fumador, } \\
\text { exfumador, } \\
\text { nunca fumador }\end{array}$ & $35-64 / \geq 65$ & CPSII & 1998 & 1989 & - & $\begin{array}{l}\text { Tuberculosis, } \\
\text { neumonía e } \\
\text { influenza y asma }\end{array}$ \\
\hline $\begin{array}{l}\text { Banegas } \\
\text { et al., } 2003\end{array}$ & España & - & $\geq 35$ años & - & 1987 & - & - & CPS II & 1995-1999 & 1989 & - & $\begin{array}{c}\text { Enfermedad } \\
\text { hipertensiva, } \\
\text { tuberculosis, } \\
\text { neumonía e } \\
\text { influenza y asma }\end{array}$ \\
\hline $\begin{array}{l}\text { Montes } \\
\text { et al., } 2004\end{array}$ & España & 2001 & $\geq 35$ años & $\begin{array}{c}\text { No } \\
\text { especifican }\end{array}$ & 2001 & $\begin{array}{l}\text { Fumador, } \\
\text { exfumador, } \\
\text { nunca fumador }\end{array}$ & $35-64 / \geq 65$ & CPS॥ & 2001 & 1989 & - & $\begin{array}{l}\text { Enfermedad } \\
\text { hipertensiva, } \\
\text { neumonía y } \\
\text { gripe }\end{array}$ \\
\hline $\begin{array}{l}\text { Banegas } \\
\text { et al., } 2005\end{array}$ & España & 2001 & $\geq 35$ años & $35-64 / \geq 65$ & 2001 & $\begin{array}{l}\text { Fumador, } \\
\text { exfumador, } \\
\text { nunca fumador }\end{array}$ & $35-64 / \geq 65$ & CPS II & 2001 & 2004 & - & Varias $\S$ \\
\hline $\begin{array}{l}\text { Hernández } \\
\text { García } \\
\text { et al., } 2010\end{array}$ & España & 2006 & $\geq 35$ años & $\begin{array}{c}\text { No } \\
\text { especifican }\end{array}$ & 2006 & $\begin{array}{c}\text { Fumador, } \\
\text { exfumador, } \\
\text { nunca fumador }\end{array}$ & $35-64 / \geq 65$ & CPS II & 2006 & 1989 & - & $\begin{array}{c}\text { Enfermedad } \\
\text { hipertensiva, } \\
\text { neumonía, gripe, } \\
\text { tuberculosis y } \\
\text { asma }\end{array}$ \\
\hline $\begin{array}{l}\text { Banegas et al., } \\
2011\end{array}$ & España & 2006 & $\geq 35$ años & $35-64 / \geq 65$ & 2006 & $\begin{array}{l}\text { Fumador, } \\
\text { exfumador, } \\
\text { nunca fumador }\end{array}$ & $35-64 / \geq 66$ & CPS II & 2006 & 1989 & - & Varias $\S$ \\
\hline $\begin{array}{l}\text { Gutiérrez } \\
\text { Abejón } \\
\text { et al., } 2015\end{array}$ & España & 2012 & $\geq 18$ años & $\begin{array}{c}\text { No } \\
\text { especifican }\end{array}$ & 2012 & $\begin{array}{l}\text { Fumador, } \\
\text { exfumador, } \\
\text { nunca fumador }\end{array}$ & $\begin{array}{c}18-34 / 35 \\
64 / \geq 65\end{array}$ & CPS II & 2012 & 1989 & - & $\begin{array}{c}\text { Enfermedad } \\
\text { hipertensiva, } \\
\text { neumonía, gripe, } \\
\text { tuberculosis y } \\
\text { asma }\end{array}$ \\
\hline $\begin{array}{l}\text { Pérez Ríos } \\
\text { et al., } 2020\end{array}$ & España & 2016 & $\geq 35$ años & $\begin{array}{c}35-54 / \\
55-64 / \\
65-74 / \geq 75\end{array}$ & $\begin{array}{l}2014 y \\
2016\end{array}$ & $\begin{array}{l}\text { Fumador, } \\
\text { exfumador, } \\
\text { nunca fumador }\end{array}$ & $\begin{array}{c}35-54 / 55- \\
64 / 65-74 / \\
\geq 75\end{array}$ & 5 cohortes $* *$ & 2016 & 2014 & - & - \\
\hline Rey et al., 2021 & $\begin{array}{l}\text { España/ } \\
17 \text { CCAA }\end{array}$ & 2017 & $\geq 35$ años & $\begin{array}{c}35-54 / \\
55-64 / \\
65-74 / \geq 75\end{array}$ & $\begin{array}{l}2011,2014 \\
\text { y } 2016\end{array}$ & $\begin{array}{c}\text { Fumador, } \\
\text { exfumador, } \\
\text { nunca fumador }\end{array}$ & $\begin{array}{c}35-54 / 55- \\
64 / 65-74 / \\
\geq 75\end{array}$ & 5 cohortes $^{* *}$ & 2017 & 2014 & - & - \\
\hline \multicolumn{13}{|c|}{ COMUNIDAD AUTÓNOMA, PROVINCIA, CIUDAD } \\
\hline $\begin{array}{l}\text { Rodríguez } \\
\text { Tapioles } \\
\text { et al., } 1997\end{array}$ & Granada & 1985 & $\geq 15$ años & $\begin{array}{c}\leq 45 / 46-65 / \\
66-75 / \geq 76\end{array}$ & 1990 & - & - & Metaanálisis & 1985 & 1989 & $\begin{array}{l}\text { Cáncer cérvix, } \\
\text { enfermedad } \\
\text { reumática, } \\
\text { cardiopulmonar }\end{array}$ & . \\
\hline $\begin{array}{l}\text { Valero Juan } \\
\text { et al., } 1999\end{array}$ & Extremadura & 1993 & $\geq 15$ años & - & 1993 & $\begin{array}{l}\text { Fumador, } \\
\text { exfumador, } \\
\text { nunca fumador }\end{array}$ & - & $\begin{array}{l}\text { Estudio de } \\
\text { Granada } \\
\text { (Rodríguez } \\
\text { Tapioles et al., } \\
\text { 1994) }\end{array}$ & 1993 & 1989 & $\begin{array}{l}\text { Enfermedad } \\
\text { reumática, } \\
\text { cardiopulmonar, } \\
\text { aneurisma } \\
\text { aórtico, } \\
\text { aterosclerosis y } \\
\text { otras vasculares }\end{array}$ & , \\
\hline $\begin{array}{l}\text { Bello Luján } \\
\text { et al., } 2001\end{array}$ & $\begin{array}{l}\text { Islas } \\
\text { Canarias }\end{array}$ & $\begin{array}{l}1975- \\
1994\end{array}$ & $\geq 35$ años & $\begin{array}{c}\text { No } \\
\text { especifican }\end{array}$ & 1975-1994 & $\begin{array}{l}\text { Fumador, } \\
\text { exfumador, } \\
\text { nunca fumador }\end{array}$ & $\begin{array}{c}35-44 / 45 \\
64 / \geq 65\end{array}$ & CPS II & $1975-1994$ & 1989 & - & $\begin{array}{l}\text { Enfermedad } \\
\text { hipertensiva, } \\
\text { neumonía } \\
\text { e influenza, } \\
\text { tuberculosis y } \\
\text { asma }\end{array}$ \\
\hline
\end{tabular}


Tabla 1. (Cont.)

\begin{tabular}{|c|c|c|c|c|c|c|c|c|c|c|c|c|}
\hline \multirow[b]{2}{*}{ Autor } & \multirow[b]{2}{*}{ Ámbito } & \multirow{2}{*}{$\begin{array}{c}\text { Año } \\
\text { estimación } \\
\text { MA }\end{array}$} & \multirow{2}{*}{$\begin{array}{c}\text { Edad } \\
\text { población }\end{array}$} & \multirow{2}{*}{$\begin{array}{l}\text { Grupos de } \\
\text { edad de la } \\
\text { estimación }\end{array}$} & \multicolumn{3}{|c|}{ Prevalencia } & \multirow{2}{*}{$\begin{array}{c}\text { Riesgo Relativo } \\
\text { Fuente }\end{array}$} & \multicolumn{4}{|c|}{ Mortalidad observada } \\
\hline & & & & & Año & $\begin{array}{l}\text { Categorías } \\
\text { consumo }\end{array}$ & $\begin{array}{l}\text { Grupos } \\
\text { de edad }\end{array}$ & & Año & Año SG $\phi$ & $\begin{array}{l}\text { Causas no } \\
\text { incluidas? }\end{array}$ & $\begin{array}{c}\text { Causas } \\
\text { adicionales }\end{array}$ \\
\hline \multicolumn{13}{|c|}{ COMUNIDAD AUTÓNOMA, PROVINCIA, CIUDAD } \\
\hline $\begin{array}{l}\text { Santana Armas } \\
\text { et al., } 1998\end{array}$ & $\begin{array}{c}\text { Islas } \\
\text { Canarias }\end{array}$ & 1995 & - & - & 1995 & - & - & CPS II & 1995 & 1989 & $\begin{array}{l}\text { Enfermedad } \\
\text { reumática, } \\
\text { cardiopulmonar, } \\
\text { aneurisma } \\
\text { aórtico, } \\
\text { aterosclerosis y } \\
\text { otras vasculares† }\end{array}$ & $\begin{array}{c}\text { Enfermedad } \\
\text { hipertensiva y } \\
\text { asma }\end{array}$ \\
\hline $\begin{array}{l}\text { Santos Zarza } \\
\text { et al., } 2001\end{array}$ & $\begin{array}{l}\text { Castilla y } \\
\text { León }\end{array}$ & 1995 & $\geq 15$ años & - & 1993 & $\begin{array}{l}\text { Fumador, } \\
\text { exfumador, } \\
\text { nunca fumador }\end{array}$ & - & $\begin{array}{l}\text { Estudio de } \\
\text { Granada } \\
\text { (Rodríguez } \\
\text { Tapioles et al., } \\
\text { 1994) }\end{array}$ & 1995 & 1989 & $\begin{array}{l}\text { Enfermedad } \\
\text { reumática, } \\
\text { cardiopulmonar, } \\
\text { aneurisma } \\
\text { aórtico, } \\
\text { aterosclerosis y } \\
\text { otras vascularest }\end{array}$ & t \\
\hline $\begin{array}{l}\text { Jané et al., } \\
2003\end{array}$ & Barcelona & 1983-1998 & $\geq 35$ años & $\begin{array}{c}35-44 / 45-54 \\
/ 55-64 / 65- \\
74 / \geq 75\end{array}$ & 1983-1998 & - & - & CPS II & 1983-1998 & 1989 & - & $\begin{array}{c}\text { Enfermedad } \\
\text { hipertensiva, } \\
\text { neumonía e } \\
\text { influenza y asma }\end{array}$ \\
\hline $\begin{array}{l}\text { Zorrilla Torras et } \\
\text { al., } 2005\end{array}$ & Madrid & $1992-1998$ & $\geq 35$ años & $35-64 / \geq 65$ & 1992-1998 & $\begin{array}{c}\text { Fumador, } \\
\text { exfumador, } \\
\text { nunca fumador }\end{array}$ & - & CPS II & - & 1989 & - & $\begin{array}{l}\text { Enfermedad } \\
\text { hipertensiva, } \\
\text { neumonía } \\
\text { e influenza, } \\
\text { tuberculosis y } \\
\text { asma }\end{array}$ \\
\hline $\begin{array}{l}\text { Pérez Ríos } \\
\text { et al., } 2009\end{array}$ & Galicia & $2001-2006$ & $\geq 35$ años & $35-64 / \geq 65$ & 2001-2006 & $\begin{array}{c}\text { Fumador, } \\
\text { exfumador, } \\
\text { nunca fumador }\end{array}$ & $35-64 / \geq 65$ & CPS II & - & 2004 & - & - \\
\hline $\begin{array}{l}\text { Pérez Ríos } \\
\text { et al., } 2011\end{array}$ & Galicia & $1980-2007$ & $\geq 35$ años & $35-64 / \geq 65$ & No procede* & No procede* & No procede* & CPS II & $1980-2007$ & 2004 & - & - \\
\hline
\end{tabular}

Nota. CPS II: Cancer Prevention Study II, SG: Surgeon General.

$\phi$ Año del informe de Surgeon General en el que se basó el estudio para establecer las causas de muerte asociadas al consumo de tabaco.

II Incluido en relación con las causas establecidas por el Surgeon General. Se considera que las causas son las enfermedades con las que se ha establecido una relación causal, y estas últimas corresponden a entidades nosológicas individuales (p. Ej., Cáncer de estómago) o grupos de códigos de la 10a Clasificación Internacional de Enfermedades (CIE -10) utilizados por la convención internacional (p. Ej., Pulmón se refiere a tráquea, bronquios y pulmón).

† Dentro de "otras vasculares" se incluyen las siguientes enfermedades según códigos CIE-10: I72 (otros tipos de aneurisma), I73 (otras enfermedades vasculares periféricas), I74 (embolia y trombosis arterial), I75 (ateroembolismo), I76 (embolia arterial séptica), I77 (otros trastornos de arterias y arteriolas), I78 (enfermedad de los capilares).

§ Incluye según códigos CIE-10: C46.2, C45.7, C68, I10-I15, I52, I97-I98, Roo.1, Roo.8, Ro1.2, G45, G93.6, G93.8, G95.1, M30-M31, J20.9, J98.o, A15-A16, A48.1, Bo5.2, B90.9, J45-J46, 165

* Método de estimación empleado: independiente de prevalencias.

** The National Institutes of Health- American Association of Retired Persons Diet and Health Study, the American Cancer Society’s CPS II Nutrition Cohort, the Women's Health Initiative, the Nurses' Health Study y the Health Professionals Follow-up Study.

zález Enríquez et al., 1997), hasta las últimas (Pérez-Ríos et al., 2020; Rey et al., 2021).

En España, en relación con las causas específicas, la cardiopatía isquémica fue reemplazada por el cáncer de pulmón como principal causa específica de MA al tabaco entre los hombres en 1992 (González Enríquez et al., 1997). En las mujeres destaca que la cardiopatía isquémica primero fue reemplazada por la enfermedad pulmonar obstructiva crónica (EPOC) en 1992 (González Enríquez et al., 1997) y ésta última por el cáncer de pulmón en 2006 (Banegas et al., 2011). En la Tabla 2 se proporciona un resumen detallado de los principales resultados de todos los estudios incluidos.

\section{Discusión}

Este trabajo pone de manifiesto que en España las estimaciones de MA no se realizan de forma periódica y que tampoco se dispone de información comparable que permita valorar el impacto que tiene el consumo de tabaco en niveles territoriales más desagregados como las CCAA. A pesar de que los datos apuntan a variaciones en la evolución de la epidemia tabáquica en términos de prevalencia en los diferentes territorios de España, los estudios que estiman MA a un nivel más desagregado, es decir, por CCAA, provincias o ciudades, son escasos y no permiten extraer conclusiones al respecto del impacto sobre la mortalidad. 
Julia Rey-Brandariz, Mónica Pérez-Ríos, Maria Isolina Santiago-Pérez, Leonor Varela-Lema, Alexandra Giraldo-Osorio, Nerea Mourino, Alberto Ruano-Ravina

Tabla 2. Porcentajes de mortalidad atribuida (MA) al consumo de tabaco sobre la mortalidad observada y grupo de causas de muerte y causa especifica de muerte con mayor carga de MA al consumo de tabaco. Los datos se presentaron en total (hombres y mujeres), en hombres y en mujeres.

\begin{tabular}{|c|c|c|c|c|c|c|c|c|c|c|c|}
\hline \multirow{2}{*}{ Autor } & \multirow{2}{*}{ Ámbito } & \multirow{2}{*}{$\begin{array}{l}\text { Año } \\
\text { estimación } \\
\text { MA }\end{array}$} & \multicolumn{3}{|c|}{ Porcentaje de MA } & \multicolumn{3}{|c|}{$\begin{array}{c}\text { Grupo de causas de muerte con } \\
\text { más MA }\end{array}$} & \multicolumn{3}{|c|}{ Causa específica de muerte con más MA } \\
\hline & & & Total & Hombres & Mujeres & Total & Hombres & Mujeres & Total & Hombres & Mujeres \\
\hline \multicolumn{12}{|c|}{ NACIONAL } \\
\hline $\begin{array}{l}\text { González } \\
\text { Enríquez } \\
\text { et al., 1989a }\end{array}$ & España & 1983 & $13,0 \%$ & $20,3 \%$ & $5,4 \%$ & ECV & ECV & ECV & $\begin{array}{l}\text { Cardiopatía } \\
\text { isquémica }\end{array}$ & $\begin{array}{l}\text { Cardiopatía } \\
\text { isquémica }\end{array}$ & $\begin{array}{l}\text { Cardiopatía } \\
\text { isquémica }\end{array}$ \\
\hline $\begin{array}{l}\text { González } \\
\text { Enríquez } \\
\text { et al., 1989b }\end{array}$ & España & 1984 & $13,8 \%$ & - & - & ECV & ECV & ECV & $\begin{array}{l}\text { Cardiopatía } \\
\text { isquémica }\end{array}$ & $\begin{array}{l}\text { Cardiopatía } \\
\text { isquémica }\end{array}$ & $\begin{array}{l}\text { Cardiopatía } \\
\text { isquémica }\end{array}$ \\
\hline $\begin{array}{l}\text { González } \\
\text { Enríquez } \\
\text { et al., } 1997\end{array}$ & España & $\begin{array}{l}1978- \\
1992\end{array}$ & $\begin{array}{l}\text { 1978: } 13,7 \% \\
\text { 1992: } 14,7 \%\end{array}$ & $\begin{array}{l}1992: \\
26,3 \%\end{array}$ & $\begin{array}{l}1992: \\
2,0 \%\end{array}$ & $\begin{array}{l}\text { 1978: ECV } \\
\text { 1992: } \\
\text { tumores }\end{array}$ & $\begin{array}{l}\text { 1978: ECV } \\
\text { 1992: } \\
\text { tumores }\end{array}$ & $\begin{array}{l}\text { 1978: ECV } \\
\text { 1992: ECV }\end{array}$ & $\begin{array}{l}\text { 1978: ACV 1992: } \\
\text { cáncer de } \\
\text { pulmón }\end{array}$ & $\begin{array}{l}\text { 1978: ACV 1992: } \\
\text { cáncer de } \\
\text { pulmón }\end{array}$ & $\begin{array}{l}\text { 1978: ACV } \\
\text { 1992: EPOC }\end{array}$ \\
\hline $\begin{array}{l}\text { Banegas } \\
\text { et al., } 2001\end{array}$ & España & 1998 & $16,0 \%$ & $28,3 \%$ & $2,5 \%$ & Tumores & Tumores & ECV & $\begin{array}{l}\text { Cáncer de } \\
\text { pulmón }\end{array}$ & $\begin{array}{l}\text { Cáncer de } \\
\text { pulmón }\end{array}$ & EPOC \\
\hline $\begin{array}{l}\text { Banegas } \\
\text { et al., } 2003\end{array}$ & España & - & $16,0 \%$ & - & - & Tumores & Tumores & ECV & $\begin{array}{l}\text { Cáncer de } \\
\text { pulmón }\end{array}$ & $\begin{array}{l}\text { Cáncer de } \\
\text { pulmón }\end{array}$ & EPOC \\
\hline $\begin{array}{l}\text { Montes } \\
\text { et al., } 2004\end{array}$ & España & 2001 & - & $24,5 \%$ & $2,6 \%$ & Tumores & Tumores & ECV & $\begin{array}{l}\text { Cáncer de } \\
\text { pulmón }\end{array}$ & $\begin{array}{l}\text { Cáncer de } \\
\text { pulmón }\end{array}$ & EPOC \\
\hline $\begin{array}{l}\text { Banegas } \\
\text { et al., } 2005\end{array}$ & España & 2001 & $15,5 \%$ & $27,1 \%$ & $2,9 \%$ & Tumores & Tumores & ECV & $\begin{array}{l}\text { Cáncer de } \\
\text { pulmón }\end{array}$ & $\begin{array}{l}\text { Cáncer de } \\
\text { pulmón }\end{array}$ & EPOC \\
\hline $\begin{array}{l}\text { Hernández } \\
\text { García } \\
\text { et al., } 2010\end{array}$ & España & 2006 & $16,2 \%$ & $23,9 \%$ & $7,8 \%$ & Tumores & Tumores & ECV & $\begin{array}{l}\text { Cáncer de } \\
\text { pulmón }\end{array}$ & $\begin{array}{l}\text { Cáncer de } \\
\text { pulmón }\end{array}$ & $\begin{array}{l}\text { Otras } \\
\text { cardiopatías }\end{array}$ \\
\hline $\begin{array}{l}\text { Banegas } \\
\text { et al., } 2011\end{array}$ & España & 2006 & $14,7 \%$ & $25,1 \%$ & $3,4 \%$ & Tumores & Tumores & ECV & $\begin{array}{l}\text { Cáncer de } \\
\text { pulmón }\end{array}$ & $\begin{array}{l}\text { Cáncer de } \\
\text { pulmón }\end{array}$ & $\begin{array}{l}\text { Cáncer de } \\
\text { pulmón }\end{array}$ \\
\hline $\begin{array}{l}\text { Gutiérrez } \\
\text { Abejón } \\
\text { et al., } 2015\end{array}$ & España & 2012 & $15,2 \%$ & $22,6 \%$ & $7,6 \%$ & Tumores & Tumores & ECV & $\begin{array}{l}\text { Cáncer de } \\
\text { pulmón }\end{array}$ & $\begin{array}{l}\text { Cáncer de } \\
\text { pulmón }\end{array}$ & $\begin{array}{c}\text { Otras } \\
\text { cardiopatías }\end{array}$ \\
\hline $\begin{array}{l}\text { Pérez Ríos } \\
\text { et al., } 2020\end{array}$ & España & 2016 & $13,7 \%$ & - & - & Tumores & Tumores & Tumores & $\begin{array}{l}\text { Cáncer de } \\
\text { pulmón }\end{array}$ & $\begin{array}{l}\text { Cáncer de } \\
\text { pulmón }\end{array}$ & $\begin{array}{l}\text { Cáncer de } \\
\text { pulmón }\end{array}$ \\
\hline $\begin{array}{l}\text { Rey et al., } \\
2021\end{array}$ & $\begin{array}{l}\text { España/ } \\
17 \text { CCAA }\end{array}$ & 2017 & $12,9 \%$ & - & - & Tumores & Tumores & Tumores & $\begin{array}{l}\text { Cáncer de } \\
\text { pulmón }\end{array}$ & $\begin{array}{l}\text { Cáncer de } \\
\text { pulmón }\end{array}$ & $\begin{array}{l}\text { Cáncer de } \\
\text { pulmón }\end{array}$ \\
\hline \multicolumn{12}{|c|}{ COMUNIDAD AUTÓNOMA, PROVINCIA, CIUDAD } \\
\hline $\begin{array}{l}\text { Rodríguez } \\
\text { Tapioles } \\
\text { et al., } 1997\end{array}$ & Granada & 1985 & $15,9 \%$ & $21,2 \%$ & $10,0 \%$ & ECV & ECV & ECV & $\begin{array}{l}\text { Cardiopatía } \\
\text { isquémica }\end{array}$ & EPOC & $\begin{array}{l}\text { Cardiopatía } \\
\text { isquémica }\end{array}$ \\
\hline $\begin{array}{l}\text { Valero Juan } \\
\text { et al., } 1999\end{array}$ & Extremadura & 1993 & $11,7 \%$ & $16,8 \%$ & $6,3 \%$ & Tumores & Tumores & ECV & $\begin{array}{l}\text { Cáncer de } \\
\text { pulmón }\end{array}$ & $\begin{array}{l}\text { Cáncer de } \\
\text { pulmón }\end{array}$ & ACV \\
\hline $\begin{array}{l}\text { Bello Luján } \\
\text { et al., } 2001\end{array}$ & Islas Canarias & $1975-1994$ & $\begin{array}{l}1975: 20,0 \% \\
1994: 15,3 \%\end{array}$ & - & - & $\begin{array}{l}\text { 1975: ECV } \\
\text { 1994: ECV }\end{array}$ & $\begin{array}{l}\text { 1975: ECV } \\
\text { 1994: ECV }\end{array}$ & $\begin{array}{l}\text { 1975: ECV } \\
\text { 1994: ECV }\end{array}$ & $\begin{array}{l}\text { 1975: ACV 1994: } \\
\text { cardiopatía } \\
\text { isquémica }\end{array}$ & $\begin{array}{l}\text { 1975: ACV 1994: } \\
\text { cáncer de } \\
\text { pulmón }\end{array}$ & $\begin{array}{l}\text { 1975: ACV 1994: } \\
\text { cardiopatía } \\
\text { isquémica }\end{array}$ \\
\hline $\begin{array}{l}\text { Santana } \\
\text { Armas } \\
\text { et al., } 1998\end{array}$ & Islas Canarias & 1995 & $14,9 \%$ & $20,2 \%$ & $8,0 \%$ & Tumores & - & - & $\begin{array}{l}\text { Cáncer de } \\
\text { pulmón }\end{array}$ & $\begin{array}{l}\text { Cáncer de } \\
\text { pulmón }\end{array}$ & $\begin{array}{l}\text { Cáncer de } \\
\text { pulmón }\end{array}$ \\
\hline $\begin{array}{l}\text { Santos Zarza } \\
\text { et al., } 2001\end{array}$ & Castilla y León & 1995 & $9,4 \%$ & $7,2 \%$ & $2,3 \%$ & ECV & ECV & ECV & $\begin{array}{l}\text { Cáncer de } \\
\text { pulmón }\end{array}$ & $\begin{array}{l}\text { Cáncer de } \\
\text { pulmón }\end{array}$ & $\begin{array}{l}\text { Cardiopatía } \\
\text { isquémica }\end{array}$ \\
\hline $\begin{array}{l}\text { Criado Álvarez } \\
\text { et al., } 2002\end{array}$ & $\begin{array}{l}\text { Castilla La } \\
\text { Mancha }\end{array}$ & 1987 y 1997 & $\begin{array}{l}1987: 18,7 \% \\
1997: 18,7 \%\end{array}$ & $\begin{array}{l}\text { 1987: } 14,2 \% \\
1997: 15,0 \%\end{array}$ & $\begin{array}{l}1987: 4,5 \% \\
1997: 3,8 \%\end{array}$ & $\begin{array}{l}\text { 1987: ECV } \\
\text { 1997: ECV }\end{array}$ & - & - & $\begin{array}{l}\text { 1987: ACV 1997: } \\
\text { cáncer de pulmón }\end{array}$ & $\begin{array}{l}\text { 1987: Cáncer de } \\
\text { pulmón 1997: } \\
\text { Cáncer de pulmón }\end{array}$ & $\begin{array}{c}\text { 1987: Otras } \\
\text { cardiopatías 1997: } \\
\text { Otras cardiopatías }\end{array}$ \\
\hline $\begin{array}{l}\text { Jané et al., } \\
2003\end{array}$ & Barcelona & 1983-1998 & $\begin{array}{c}1983: 9,6 \% \\
1998: 13,8 \%\end{array}$ & $\begin{array}{l}1998: \\
25,1 \%\end{array}$ & $\begin{array}{l}1998: \\
2,9 \%\end{array}$ & $\begin{array}{l}\text { 1983: } \\
\text { ECV 1998: } \\
\text { Tumores }\end{array}$ & $\begin{array}{l}\text { 1983: } \\
\text { ECV 1998: } \\
\text { Tumores }\end{array}$ & $\begin{array}{l}\text { 1983: ECV } \\
\text { 1998: ECV }\end{array}$ & $\begin{array}{l}\text { 1998: cáncer de } \\
\text { pulmón }\end{array}$ & $\begin{array}{l}\text { Cáncer de } \\
\text { pulmón }\end{array}$ & EPOC \\
\hline $\begin{array}{l}\text { Zorrilla Torras } \\
\text { et al., } 2005\end{array}$ & Madrid & 1992-1998 & 1998: $15,9 \%$ & $\begin{array}{l}1998: \\
28,4 \%\end{array}$ & $\begin{array}{l}\text { 1998: } \\
2,8 \%\end{array}$ & $\begin{array}{l}\text { 1998: } \\
\text { Tumores }\end{array}$ & $\begin{array}{l}\text { 1998: } \\
\text { Tumores }\end{array}$ & 1998: ECV & $\begin{array}{l}\text { 1998: Cáncer de } \\
\text { pulmón }\end{array}$ & $\begin{array}{l}\text { 1998: Cáncer de } \\
\text { pulmón }\end{array}$ & $\begin{array}{l}\text { 1998: Cáncer de } \\
\text { pulmón }\end{array}$ \\
\hline $\begin{array}{l}\text { Pérez Ríos et } \\
\text { al., } 2009\end{array}$ & Galicia & $2001-2006$ & $12,5 \%$ & $22,4 \%$ & $2,2 \%$ & Tumores & Tumores & Tumores & $\begin{array}{l}\text { Cáncer de } \\
\text { pulmón }\end{array}$ & $\begin{array}{l}\text { Cáncer de } \\
\text { pulmón }\end{array}$ & EPOC \\
\hline $\begin{array}{l}\text { Pérez Ríos et } \\
\text { al., } 2011\end{array}$ & Galicia & $1980-2007$ & $12,6 \%$ & $23,5 \%$ & $1,5 \%$ & Tumores & Tumores & $\begin{array}{l}\text { ECVy } \\
\text { enfermedad } \\
\text { respiratoria }\end{array}$ & $\begin{array}{l}\text { Cáncer de } \\
\text { pulmón }\end{array}$ & $\begin{array}{l}\text { Cáncer de } \\
\text { pulmón }\end{array}$ & - \\
\hline
\end{tabular}

Nota. ACV: accidente cerebrovascular. ECV: enfermedad cardiovascular. EPOC: enfermedad pulmonar obstructiva crónica. MA: mortalidad atribuida. 
Todos los estudios que estiman MA al consumo de tabaco en España emplean datos de MO que proceden de la estadística de defunciones por causa de muerte que gestiona el INE, pero no siempre incluyen todas las patologías con relación causal establecida con el consumo de tabaco en el momento de la estimación en los informes del Surgeon General. El Surgeon General ha publicado 4 informes principales en los que se evalúa el estado de las asociaciones causales entre consumo de tabaco y mortalidad. En el primero, publicado en 1964, se estableció relación causal con el cáncer de pulmón y de laringe en hombres y la bronquitis crónica (U.S. Department of Health, Education and Welfare 1964). El segundo informe, publicado en 1989, incluyó las relaciones causales con varios tumores (pulmón y laringe en ambos sexos, labio, cavidad oral, faringe, esófago, páncreas, cuello de útero, vejiga, riñón y pelvis renal); enfermedades cardiovasculares (cardiopatía isquémica, enfermedad cerebrovascular, enfermedad reumática del corazón, enfermedad cardiopulmonar, aterosclerosis, aneurisma aórtico y otras vasculares (clasificadas según CIE-10 como I72-I78)) (U.S. Department of Health and Human Services, 1989). En el tercer informe, publicado en 2004, se añadieron las relaciones causales de cáncer de estómago, leucemia mieloide aguda y neumonía e influenza (U.S. Department of Health and Human Services, 2004). En el último informe publicado en 2014, se establecieron como nuevas relaciones causales el cáncer colorrectal, cáncer de células hepáticas, tuberculosis y diabetes mellitus. En la actualidad, diferentes causas de muerte como el cáncer de mama continúan en estudio (U.S. Department of Health and Human Services, 2014). Alguno de los estudios de estimación realizados en España o no incluyeron todas las patologías con relación causal establecida en el momento de realización (González Enríquez et al., 1997; Rodriguez Tapioles et al., 1997; Santana Armas et al., 1998; Santos Zarza et al., 2001; Valero Juan et al., 1999) o incluyeron más causas (Banegas et al., 2001; Banegas et al., 2003; Banegas et al., 2005; Banegas et al., 2011; Bello Luján et al., 2001; Criado-Álvarez et al., 2002; González Enríquez et al., 1989a; González Enríquez et al., 1989b; González Enríquez et al., 1997; Gutiérrez-Abejón et al., 2015; Hernández-García et al., 2010; Jané et al., 2003; Montes et al., 2004; Santana Armas et al., 1998; Zorrilla-Torras et al., 2005). Esto último puede ser debido a que varios estudios incorporan patologías sobre las que la evidencia disponible sugería que había una asociación con el consumo de tabaco, sin embargo, no había suficiente evidencia para establecer una relación causal en ese momento.

A día de hoy, no se ha establecido la edad a partir de la que se debe atribuir la mortalidad al consumo de tabaco, aunque la mayoría de los estudios incluyen como límite inferior de edad los 35 años. Estimar la MA en edades tempranas entra en conflicto con el criterio de causalidad de precedencia temporal, según el cual un factor de riesgo causa enfermedad o muerte después de estar expuesto a él durante el tiempo suficiente para que produzca daños. Esta limitación también se puede aplicar a los datos de prevalencia, ya que en varios estudios la prevalencia empleada es temporalmente próxima a la $\mathrm{MO}$ y no tiene en cuenta el lapso de tiempo entre la exposición y el efecto. Disponer de una guía metodológica, basada por ejemplo en las guías STROBE, permitiría mejorar la comunicación y homogeneizaría la publicación de los resultados, facilitando la realización de estos análisis.

Desde la primera estimación de MA al consumo de tabaco en 1978, diferentes estudios realizados a nivel nacional reflejan un aumento en la carga de MA al consumo de tabaco hasta el año 2001, momento en el que se observa por primera vez un descenso en los hombres. La comparación directa de las estimaciones es complicada debido a las diferencias entre estudios en cuanto a los cambios en la estructura etaria de la población desde 1978 hasta 2017, los cambios en las causas estudiadas o los riesgos empleados. Si bien, destaca que al comparar la MA en los hombres y en las mujeres, se observa un aumento de la MA en las mujeres en comparación con los hombres, así, en las últimas estimaciones de 2016 y 2017, alrededor del $84 \%-85 \%$ de las muertes atribuidas al tabaco ocurrieron en hombres (Pérez-Ríos et al., 2020; Rey et al., 2021) frente al 96,6\% que se observaba en la primera estimación en 1978 (González Enríquez et al., 1997). En países como Estados Unidos de América (EEUU) o Reino Unido donde la epidemia tabáquica está más evolucionada, se empezó a observar el aumento de las cifras de MA en las mujeres décadas antes de verse en las mujeres españolas. En el caso de EEUU, el impacto del consumo de tabaco en la mortalidad femenina empezó a observarse hacia finales de los años 50 y desde entonces las cifras de MA han ido aumentando hasta llegar a igualarse a las masculinas en el periodo 2005-2010 (Peto, Lopez, Boreham y Thun, 2011). En el caso de Reino Unido, el aumento de MA al consumo de tabaco en mujeres comenzó antes de 1950 y, al igual que en EEUU, este porcentaje continuó ascendiendo hasta prácticamente igualarse con los hombres entre 2005-2010 (Peto et al., 2011). En España, en comparación con Reino Unido y EEUU, las mujeres se incorporaron más tarde al consumo de tabaco y el descenso que se observa en la prevalencia de consumo en los hombres desde 1987, no se ha observado en las mujeres hasta 2006. Así, en los hombres, en el periodo 1987-2005, el descenso absoluto promedio anual de la prevalencia en fumadores fue del 1\% y en el periodo 2006-2014 del 0,7\%. En las mujeres el descenso promedio anual en el periodo 2006-2014 fue del 0,5\% (Fernández et al., 2017). Esto hace que hombres y mujeres se sitúen en dos fases diferentes del modelo de evolución de epidemia tabáquica. Los hombres se sitúan en la fase IV caracterizada por el descenso en la prevalencia de consumo y de la MA al tabaco entre la población de 35 a 69 años, y las mujeres aún podrían situar- 
se en una fase III tardía caracterizada por la estabilización de la prevalencia y aumento de la MA (Lopez, Collishaw y Piha, 1994; Thun, Peto, Boreham y Lopez, 2012).

A lo largo de los años, se ha observado un descenso en las muertes por enfermedades cardiovasculares/cardiometabólicas atribuidas al consumo de tabaco. Esto puede explicarse tanto por un mejor control de los principales factores de riesgo de las enfermedades cardiovasculares como la hipertensión, la hipercolesterolemia y el sedentarismo, como por el desarrollo de nuevos tratamientos médicos (Flores-Mateo et al., 2011). Este descenso de la MA por enfermedades cardiovasculares/cardiometabólicas ha posicionado a los tumores como el grupo de causas al que se atribuye mayor carga de mortalidad. Para interpretar estos cambios hay que considerar las grandes diferencias en el tiempo que transcurre entre la exposición al factor de riesgo y el desenlace en estos grupos de enfermedades, mucho más largo en el caso de los tumores, que en las enfermedades cardiovasculares/cardiometabólicas donde los efectos se observan en un plazo de tiempo más corto.

En relación con las causas específicas de muerte, en los hombres se observa como el cáncer de pulmón se posiciona como la principal causa de muerte por consumo de tabaco desde principios de los años 90 (González Enríquez et al., 1997). En las mujeres, el aumento del número de muertes por cáncer de pulmón ha sido más lento y no ha sido hasta las últimas estimaciones de 2016, 2017 y las realizadas en el quinquenio 2010-2014 cuando el cáncer de pulmón se posicionó como primera causa de muerte asociada al consumo de tabaco en las mujeres españolas (Ministerio de Sanidad, Consumo y Bienestar Social, 2016; Pérez-Ríos et al., 2020; Rey et al., 2021). Sin embargo, en países como EEUU y Canadá, donde el consumo de tabaco entre las mujeres empezó antes, el cáncer de pulmón ya se había posicionado como principal causa de muerte en las mujeres en los años 1990 y 1991, respectivamente (Centers for Disease Control and Prevention, 1993; Illing y Kaiserman, 1995).

La limitación más importante de este trabajo está ligada a la dificultad para, en ocasiones, resumir la información de los diferentes trabajos incluidos, ya que son muy heterogéneos en la presentación de sus resultados. Como ventajas destaca el propio diseño de revisión sistemática y la exhaustividad en la recogida de información.

En conclusión, en España se dispone de diferentes estimaciones de MA a nivel nacional, pero no tienen periodicidad regular. Aunque la evolución de la epidemia tabáquica en términos de prevalencia de consumo es diferente en las unidades territoriales que forman España, existen pocos estudios en los que se valore el diferente impacto que el consumo de tabaco tiene en la mortalidad de las unidades territoriales más pequeñas como CCAA, provincias o ciudades. La edad a partir de la cual se estima la MA, las fuentes de datos o las causas para las que se estima mor- talidad no son homogéneas entre los estudios. La actualización de las causas de mortalidad asociadas al consumo de tabaco o el valor puntual del exceso de riesgo de morir que tienen los fumadores y exfumadores, con respeto a los nunca fumadores, dificultan una valoración precisa de los cambios en las estimaciones de MA al consumo de tabaco. En general, se puede afirmar que la MA al consumo de tabaco aumentó en las mujeres españolas desde las primeras estimaciones hasta las más recientes, mientras que, en los hombres, descendió a partir de 2001. En España, actualmente, el cáncer de pulmón es la causa a la que se asocia una mayor MA al consumo de tabaco en ambos sexos. Disponer de estimaciones periódicas globales y a nivel desagregado, como por ejemplo CCAA, sería necesario para monitorizar de forma correcta la epidemia tabáquica en España y valorar el impacto de las diferentes medidas de control de tabaquismo, tanto clínicas como legislativas, que se han implementado en España en los últimos años. A pesar de que en esta revisión se observa que en global la MA al consumo de tabaco está descendiendo en España, las estimaciones más recientes aun sitúan al consumo de tabaco como el factor de riesgo que más muerte causa. Es indiscutible la necesidad de implementar medidas integrales de control de tabaquismo y medidas de prevención primaria y secundaria que permitan avanzar hacia una sociedad libre de tabaco.

\section{Conflicto de intereses}

Todos los autores declaran no tener conflictos de intereses.

\section{Referencias}

Banegas, J. R., Díez-Gañán, L., Bañuelos-Marco, B., González-Enríquez, J., Villar-Álvarez, F., Martín-Moreno, J. M.,... Jiménez-Ruiz, C. (2011). Smoking-attributable deaths in Spain, 2006. Medicina Clinica, 136, 97-102. doi:10.1016/j.medcli.2010.03.039.

Banegas, J. R., Díez Gañán, L., González Enríquez, J., Villar Álvarez, F. y Rodríguez-Artalejo, F. (2005). La mortalidad atribuible al tabaquismo comienza a descender en España. Medicina Clínica, 124, 769-771. doi:10.1157/13075847.

Banegas, J. R. B., Díez Gañán, L., Rodríguez-Artalejo, F., Pérez-Regadera, A. G. y Villar Álvarez, F. (2001). Smoking-attributable deaths in Spain in 1998. Medicina Clínica, 117, 692-694. doi:10.1016/S0025-7753(01)72226-9.

Banegas, J. R., Rodríguez-Artalejo, F., Graciani, A., Villar, F. y Herruzo, R. (2003). Mortality attributable to cardiovascular risk factors in Spain. European Journal of Clinical Nutrition, 57 (Supl. 1), S18-S21. doi:10.1038/ sj.ejcn.1601804. 
Banegas Banegas, J. R., Rodríguez Artalejo, F., Martín-Moreno, J. M., González Enríquez, J., Villar Alvarez, F. y Guasch Aguilar, A. (1993). Proyección del impacto del hábito tabáquico sobre la salud de la población española y de los beneficios potenciales de su control. Medicina Clínica, 101, 644-649.

Bello Luján, L., Lorenzo Ruano, P., Gil Muñoz, M., Saavedra Santana, P. y Serra Majem, L. (2001). Evolución de la mortalidad atribuible al tabaco en las Islas Canarias (1975-1994). Revista Española de Salud Pública, 75, 71-80.

Centers for Disease Control and Prevention. (1993). Cigarette smoking-attributable mortality and years of potential life lost-United States, 1990. Morbidity and Mortality Weekly Report, 42, 645-649.

Criado-Álvarez, J. J., Morant Ginestar, C. y De Lucas Veguillas, A. (2002). Mortalidad atribuible al consumo de tabaco en los años 1987 y 1997 en Castilla La Mancha, España. Revista Española de Salud Pública, 76, 27-36.

Fernández, E., Fu, M., Galán, I., López-Medina, M. J., Martínez, C., Martínez-Sánchez, J. M.,... Sureda, F. (2017). Evolución de las políticas de control de tabaquismo en Espana (Leyes 28/2005 y 42/2010). Revisión de la evidencia. Recuperado de https://www.seepidemiologia.es/documents / dummy/V9.0\% 20-\%20Libro\%20Tabaquismo\%202017\%20-\%20Abierto\% 20Final.pdf.

Flores-Mateo, G., Grau, M., O’Flaherty, M., Ramos, R., Elosua, R., Violan-Fors, C.,... Capewell, S. (2011). Análisis de la disminución de la mortalidad por enfermedad coronaria en una población mediterránea: España 1988-2005. Revista Española de Cardiología, 64, 988-996. doi:10.1016/j.recesp.2011.05.033.

García Benavides, F. y Hernández Aguado, I. (1989). A propósito de la mortalidad prematura atribuible al tabaco. Medicina Clinica, 93, 274-275.

Gregoraci, G., van Lenthe, F. J., Artnik, B., Bopp, M., Deboosere, P., Kovács, K.,... DEMETRIQ Consortium (2017). Contribution of smoking to socioeconomic inequalities in mortality: A study of 14 European countries, 19902004. Tobacco Control, 26, 260-268. doi:10.1136/tobaccocontrol-2015-052766.

González Enríquez, J., Rodríguez Artalejo, F., Banegas Banegas, J. R. y Villar Álvarez, F. (1989a). Deaths attributed to tobacco consumption in Spain. Correcting and updating the data. Medicina Clinica, 93, 79.

González Enríquez, J., Rodríguez Artalejo, F., Martín Moreno, J., Banegas Banegas, J. R. y Villar Álvarez, F. (1989b). Deaths attributable to tobacco consumption in Spain. Medicina Clínica, 92, 15-18.

González Enríquez, J., Villar Álvarez, F., Banegas Banegas, J., Rodríguez Artalejo, F. y Martín Moreno, J. (1997). Trends in the mortality attributable to tobacco use in Spain, 1978-1992: 600,000 deaths in 15 years. Medicina Clinica, 109, 577-582.
González-Enríquez, J., Salvador-Llivina, T., López-Nicolás, A., Antón De Las Heras, E., Musin, A., Fernández, E. y Pérez-Escolano, I. (2002). Morbilidad, mortalidad y costes sanitarios evitables mediante una estrategia de tratamiento del tabaquismo en España. Gaceta Sanitaria, 16, 308-317. doi:10.1016/s0213-9111(02)71929-8.

Gutiérrez-Abejón, E., Rejas-Gutiérrez, J., Criado-Espegel, P., Campo-Ortega, E. P., Breñas-Villalón, M. T. y Martín-Sobrino, N. (2015). Smoking impact on mortality in Spain in 2012. Medicina Clínica, 145, 520-525. doi:10.1016/j. medcle.2016.04.018.

Haeberer, M., León-Gómez, I., Pérez-Gómez, B., Téllez-Plaza, M., Pérez-Ríos, M., Schiaffino, A.,... Galán, I. (2020). Social inequalities in tobacco-attributable mortality in Spain. The intersection between age, sex and educational level. PloS One, 15, e0239866. doi:10.1371/journal. pone.0239866.

Hernández-García, I., Sáenz-González, M. C. y González-Celador, R. (2010). Mortalidad atribuible al consumo de tabaco en España en el año 2006. Anales del Sistema Sanitario de Navarra, 33, 23-33. doi:10.4321/ s1137-66272010000100003.

Illing, E. y Kaiserman, M. (1995). Mortality attributable to tobacco use in Canada and its regions, 1991. Canadian Journal of Public Health, 86, 257-265. doi:10.1093/ $\mathrm{PCH} / 6.2 .70$.

Instituto Nacional de Estadística. (2018). Defunciones según la Causa de Muerte. Recuperado de https://www.ine.es/ jaxiT3/Tabla.htm?t=7947.

Jané, M., Borrell, C., Nebot, M. y Pasarín, M. I. (2003). Impact of smoking and alcohol consumption on mortality in the population of Barcelona [Spain]: 19831998. Gaceta Sanitaria, 17, 108-115. doi:10.1016/s02139111(03)71707-5.

Janssen, F., Trias-Llimós, S. y Kunst, A. E. (2021). The combined impact of smoking, obesity and alcohol on life-expectancy trends in Europe. International Journal of Epidemiology. Avance de publicación on-line. doi:10.1093/ ije/dyaa273.

Kulik, M. C., Menvielle, G., Eikemo, T. A., Bopp, M., Jasilionis, D., Kulhánová, I., ...EURO-GBD-SE Consortium (2014). Educational inequalities in three smoking-related causes of death in 18 European populations. Nicotine and Tobacco Research, 16, 507-518. doi:10.1093/ntr/ ntt175.

Long, D., Mackenbach, J., Martikainen, P., Lundberg, O., Brønnum-Hansen, H., Bopp, M.,... Nusselder, W. (2021). Smoking and inequalities in mortality in 11 European countries: A birth cohort analysis. Population Health Metrics, 19, 3. doi:10.1186/s12963-021-00247-2.

Lopez, A. D., Collishaw, N. E. y Piha, T. (1994). A descriptive model of the cigarette epidemic in developed countries. Tobacco Control, 3, 242-247. 
Mackenbach, J. P., Kulhánová, I., Menvielle, G., Bopp, M., Borrell, C., Costa, G.,... Eurothine and EURO-GBD-SE Consortiums (2015). Trends in inequalities in premature mortality: A study of 3.2 million deaths in 13 European countries. Journal of Epidemiology and Community Health, 69, 207-206. doi:10.1136/jech-2014-204319.

Ministerio de Sanidad, Consumo y Bienestar Social. (2016). Muertes atribuibles al consumo de tabaco en España, 20002014. Madrid: Ministerio de Sanidad, Consumo y Bienestar Social. Recuperado de https:/ /www.mscbs.gob.es/ estadEstudios/estadisticas/estadisticas/estMinisterio/ mortalidad/docs/MuertesTabacoEspana2014.pdf.

Ministerio de Sanidad, Consumo y Bienestar Social. (2018). Encuesta Nacional de Salud de España 2017. Recuperado de https://www.mscbs.gob.es/estadEstudios/estadisticas/encuestaNacional/encuesta2017.htm.

Montes, A., Pérez-Ríos, M. y Gestal, J. (2004). Impacto del tabaquismo sobre la mortalidad en España. Adicciones, 16, 75-82.

Oliva-Moreno, J., Trapero-Bertran, M. y Peña-Longobardo, L. M. (2019). Gender differences in labour losses associated with smoking-related mortality. International Journal of Environmental Research and Public Health, 16, 3644. doi:10.3390/ijerph16193644.

Organización Mundial de la Salud. (2008). OMS | MPOWER un plan de medidas para hacer retroceder la epidemia de tabaquismo. Recuperado de http://www.who.int/tobacco/ mpower/package/es/.

Page, M. J., McKenzie, J. E., Bossuyt, P. M., Boutron, I., Hoffmann, T. C., Mulrow, C. D.,... Moher, D. (2021). The PRISMA 2020 statement: An update guideline for reporting systematic reviews. British Medical Journal, 372, n71. doi:10.1136/bmj.n71.

Pérez-Ríos, M. y Montes, A. (2008). Methodologies used to estimate tobacco-attributable mortality: A review. $B M C$ Public Health, 8, 22. doi:10.1186/1471-2458-8-22.

Pérez-Ríos, M., Santiago-Pérez, M. I., Caramés, S. C., De La Iglesia, B. A., Pintos, A. M. y Vidal, X. H. (2009). Mortalidad y años de esperanza de vida perdidos a causa del tabaquismo en personas mayores de 35 años en Galicia en el período 2001-2006. Revista Española de Salud Pública, 83, 557-565. doi:10.1590/s1135-57272009000400007.

Pérez-Ríos, M., Santiago-Pérez, M. I., Cerdeira-Caramés, S., Alonso, B., Seoane, B., Malvar-Pintos, A. y Hervada-Vidal, X. (2011). Mortality attributable to tobacco consumption in Galicia (Spain): 1980-2007. Medicina Clínica, 137, 247-253. doi:10.1016/j.medcli.2010.10.009.

Pérez-Ríos, M., Schiaffino, A., Montes, A., Fernández, E., López, M. J., Martínez-Sánchez, J. M.,... Galán, I. (2020). Smoking-attributable mortality in Spain in 2016. Archivos de Bronconeumología, 56, 559-563. doi:10.1016/j. arbres.2019.11.021.

Peto, R., Lopez, A.D., Boreham, J. y Thun, M. (2011). Mortality from smoking in developed countries, 1950-2010. Recu- perado de https://tobaccocontrol.bmj.com/content/ suppl/2012/02/22/tobaccocontrol-2011-050294.DC1/ tobaccocontrol-2011-050294-s1.pdf.

Rethlefsen, M. L., Kirtley, S., Waffenschmidt, S., Ayala, A. P., Moher, D., Page, M. J. y Koffel, J. B. (2021). PRISMA-S: An extension to the PRISMA statement for reporting literature searches in systematic reviews. Systematic Reviews, 10, 39. doi:10.1186/s13643-020-01542-z.

Rey, J., Pérez-Ríos, M., Santiago-Pérez, M. I., Galán, I., Schiaffino, A., Varela-Lema, L.,... Ruano-Ravina, A. (2021). Mortalidad atribuida al consumo de tabaco en las comunidades autónomas de España, 2017. Revista Española de Cardiología. Avance de publicación on-line. doi:10.1016/j.rec.2020.10.023.

Rey-Brandariz, J., Pérez-Ríos, M., Santiago-Pérez, M. I., Varela-Lema, L., Giraldo-Osorio, A., Mourino, N. y Ruano-Ravina, A. Attributable mortality to tobacco consumption in Spain: A systematic review. PROSPERO 2021 CRD42021240612. Recuperado de https:// www.crd.york.ac.uk/prospero/display_record.php?ID=CRD42021240612.

Rodríguez Tapioles, R., Bueno Cabanillas A, Pueyos Sánchez M, Espigares García, M., Martínez González, M. y Gálvez Vargas, R. (1997). Morbilidad, mortalidad y años potenciales de vida perdidos atribuibles al tabaco. Medicina Clínica, 108, 121-127.

Rodríguez Tapioles, R., Pueyos Sánchez, A., Bueno Cavanillas, A., Delgado Rodríguez, M. y Gálvez Vargas, R. (1994). Proportion of disease attributable to tobacco in the province of Granada. Medicina Clinica, 102, 571-574.

Sánchez, M. J., Payer, T., De Angelis, R., Larrañaga, N., Capocaccia, R., Martinez, C. y CIBERESP Working Group (2010). Cancer incidence and mortality in Spain: Estimates and projections for the period 1981-2012. Annals of Oncology, 21 (Supl. 3), iii30-iii36. doi:10.1093/ annonc/mdq090.

Santana Armas, J., Orengo, J., Santana Armas, Y., Lorenzo, P. y Serra Majem, L. (1998). Mortalidad atribuible al tabaquismo en Canarias. Canarias Médica, 13, 15-19.

Santos Zarza, E. F., Valero Juan, L. F. y Sáenz González, M. C. (2001). Mortality attributable to smoking in Castilla and Leon. Atención Primaria, 27, 153-158. doi:10.1016/ S0212-6567(01)78789-6.

Thun, M., Peto, R., Boreham, J. y Lopez, A. D. (2012). Stages of the cigarette epidemic on entering its second century. Tobacco Control, 21, 96-101. doi:10.1136/tobaccocontrol-2011-050294.

U. S. Department of Health, Education and Welfare. Smoking and Health. (1964). Report of the Advisory Committee to Surgeon General of the Public Health Service. Washington, DC: U. S. D. H. E. W., Centers for Disease Control and Prevention, National Center for Chronic Disease Prevention and Health Promotion. 
U. S. Department of Health and Human Services. (1989). The Health Consequences of Smoking: 25 Years of Progress. A Report of the Surgeon General. Washington, DC: U. S. D. H. H. S., Centers for Disease Control and Prevention, National Center for Chronic Disease Prevention and Health Promotion.

U. S. Department of Health and Human Services. (2004). The Health Consequences of Smoking: A Report of the Surgeon General. Atlanta, GA: U. S. D. H. H. S., Centers for Disease Control and Prevention, National Center for Chronic Disease Prevention and Health Promotion.

U. S. Department of Health and Human Services. (2014). The Health Consequences of Smoking: 50 Years of Progress. A Report of the Surgeon General. Atlanta, GA.: U. S. D. H. H. S., Centers for Disease Control and Prevention, National Center for Chronic Disease Prevention and Health Promotion.

Valero Juan, L., Carrero Santos, M., Nelia Lubián, M. y Sáenz González, M. (1999). Mortalidad atribuible al tabaco en Extremadura. Centro de Salud, 7, 250-254.

Zorrilla-Torras, B., García-Marín, N., Galán-Labaca, I. y Gandarillas-Grande, A. (2005). Smoking attributable mortality in the Community of Madrid: 1992-1998. European Journal of Public Health, 15, 43-50. doi:10.1093/ eurpub/cki110. 